\title{
El descubrimiento de oportunidades para crear valor a través del emprendimiento e innovación
}

\section{Discovering opportunities to create value through entrepreneurship and innovation}

Edison Becerra Molina. ${ }^{1}$

Recibido: 13-04-2020 / Revisado: 17-05-2020 / Aceptado: 20-06-2020 / Publicado: 03-07-2020

DOI: $\underline{\text { https://doi.org/10.33262/cienciadigital.v4i3.1305 }}$

\begin{abstract}
.
The creation of innovative ventures is one of the main factors in the economic growth of the region and the country, therefore, for these companies to live, there must be entrepreneurs with a business idea from which the companies will germinate.

For these reasons, the implementation of a theoretical-academic management model for the development of innovative entrepreneurship policies and programs at the Catholic University of Cuenca UCACUE, which is part of the author's doctoral thesis, has been considered necessary in this research work, which contributes to the training of new entrepreneurs in the different careers in correspondence with the economic-social demand of the territory of zone 6 .

Obviously, the training and development of the entrepreneurial culture of young University students, with a critical sense, promoters of participation, creatives in innovators with skills in economic analysis of financial environments, in order to create some new activity with ideas, and / or business-generating lines of employment, taking into account the most pressing needs at the local, regional and national levels.

The purpose is to promote the articulation of UCACUE with the public, community and private sector to contribute to the social fabric of Region 6, generating transfer processes and business management with added value.

It should be noted that the projects proposed for the UCACUE authorities to carry out will allow ventures in region 6 to have an impact on the generation of wealth, employment and the revitalization of the economy, influencing the benefit of society.

The model includes incubation policies, and graduation of companies, evaluation of business ideas, the creation of a monitoring and evaluation committee, indicators and evaluation criteria, articulated to the current organic law on entrepreneurship and
\end{abstract}

\footnotetext{
${ }^{1}$ Universidad Católica de Cuenca, Cuenca, Ecuador, jbecerram@ucacue.edu.ec
} 
innovation, which aims to establish the regulatory framework that encourages and encourages entrepreneurship, innovation and technological development, promoting an entrepreneurial culture and implementing new corporate and financing modalities to strengthen the entrepreneurial ecosystem.

Keywords: Education, mipyme, entrepreneurship, innovation and employability.

\section{Resumen.}

La creación de emprendimientos innovadores es uno de los talantes del crecimiento económico de la región y del país, por lo tanto, para que estas empresas vivan, deben existir emprendedores con una idea de negocio de la que posteriormente germinarán las empresas.

Por tales razones se ha considerado necesario dentro del presente trabajo investigativo la implementación de un modelo de gestión teórico-académico para el desarrollo de políticas y programas de emprendimiento innovador en la Universidad Católica de Cuenca UCACUE, que es parte de la tesis doctoral del autor, aporte a la formación de nuevos emprendedores en las diferentes carreras en correspondencia con la demanda económico- social del territorio de la zona 6.

Como es lógico, la formación y desarrollo de la cultura emprendedora de jóvenes Universitarios, con sentido crítico, promotores de la participación, creativos en innovadores con competencias de análisis económico de entornos financieros, con la finalidad de crear con ideas alguna actividad nueva, y/o líneas de negocios generadoras de empleo, tomando en cuenta las necesidades más acuciantes a nivel local, regional y nacional.

El propósito es promover la articulación de la UCACUE con el sector público, comunitario y privado para aportar al tejido social de la Región 6, generando procesos de transferencia y gestión empresarial con valor agregado.

Es conveniente destacar que los proyectos propuestos para que lleven adelante las autoridades de la UCACUE, permitirá que los emprendimientos en la región 6, incida en la generación de riqueza, empleo y la dinamización de la economía, incidiendo en beneficio a la sociedad.

El modelo incluye políticas de incubación, y graduación de empresas, evaluación de las ideas de negocio, la creación de un comité de seguimiento y evaluación, indicadores y criterios de evaluación, articulados a la actual ley orgánica de emprendimiento e innovación, que tiene como objetivo establecer el marco normativo que incentive y fomente el emprendimiento, la innovación y el desarrollo tecnológico, promoviendo la cultura emprendedora e implementando nuevas modalidades societarias y de financiamiento para fortalecer el ecosistema emprendedor.

Palabras clave: Educación, mipyme, emprendimiento, innovación y empleabilidad. 


\section{Introducción.}

El Frente a la crisis sanitaria que está afectando a todo el mundo y sobre todo el impacto nocivo en la economía de muchos países afectados gravemente por no tener medidas vigorosas para combatir el COVID-19, como es el caso del Ecuador, en donde las medidas de emergencia fueron drásticas por evitar contagios masivos, lo que aceleró por otro lado una considerable caída de negocios y comercios tanto pequeños y medianos, desencadenado por tanto el incremento del desempleo e inclusive el cierre de algunas microempresas. Bajo este contexto en la región austral y el país donde los emprendimientos de comercios han dinamizado la economía local es importante determinar nuevas estrategias que ayuden a afrontar en forma efectiva esta crisis, aplicando nuevas políticas con el afán de poder conseguir clientes, es importante también el involucramiento del sector comercial e industrial en la diversificación de productos y servicios, considerando la invención, imaginación y la creatividad, que permitirá mantener los negocios.

Por tanto, el modelo es importante por cuanto contribuirá al desarrollo de la Cultura Emprendedora en la formación de profesionales líderes y emprendedores como uno de los ejes transversales del proceso Universitario, y se encuentra definido en los siguientes términos:

Aporta a la construcción de procesos de aprendizaje y sensibilización para tomar conciencia de la importancia de los componentes fundamentales del emprendimiento y su impacto en el desarrollo del bienestar individual, familiar y social.

La generación de emprendedores es de vital importancia para el desarrollo y progreso de un país, incentivar el emprendimiento en la educación es una clave esencial para persuadir para que los alumnos asuman riesgos.

En este empeño de lograr una Universidad responsable y comprometida con la sociedad, en plena sintonía con su propósito como comunidad educativa al servicio del pueblo, deberá lograrse, paralelamente, una elevada calidad en la formación de sus profesionales y un proceso de mejora continua que esté íntimamente relacionado con la innovación y el emprendimiento de forma sustentable. De no haber innovación e integración, no resulta posible alcanzar la responsabilidad social que debe caracterizar a la Universidad.

\section{Metodología.}

En esta parte de la investigación, se utilizó para llevar a cabo el estudio, indicando el paradigma de investigación positivista, tipo de investigación diseño de campo no experimental y transeccional o transversal con apoyo documental, Según, Arias (2016) la investigación de campo o diseño de campo consiste en: La recolección de datos directamente de los sujetos investigados, o de la realidad donde ocurren los hechos, (datos primarios), sin manipular o controlar variable alguna, es decir el investigador obtiene la 
información, pero no altera las condiciones existentes. De allí su carácter de investigación no experimental (p.25).

El nivel de investigación fue la investigación descriptiva, En este sentido, es apropiado citar a Yuni y Urbano, (2006), quienes afirman que la investigación de tipo descriptiva tiene como objetivo: Llegar a conocer las situaciones, costumbres y actitudes predominantes a través de la descripción exacta de las actividades, objetos, procesos y personas. Su meta no se limita a la recolección de datos, sino a la predicción e identificación de las relaciones que existen entre dos o más variables. Los investigadores no son meros tabuladores, sino que recogen los datos sobre la base de una hipótesis o teoría, exponen y resumen la información de manera cuidadosa y luego analizan minuciosamente los resultados, a fin de extraer generalizaciones significativas que contribuyan al conocimiento (p. 33).

La población estuvo estructurada por 4 sectores escogidos, corresponde en esta oportunidad referirnos al universo de la población representada por los ejecutivos empresariales de la provincia del Azuay, considerando la población sobre una base de la Súper Intendencia de Compañías, Valores y Seguros, se tuvo 2.001 empresas, mediante muestreo estratificado se determinó la muestra $\mathrm{n}=231$, considerando la varianza muestral del 0.05 , el nivel de confianza representó el 95\% de confianza, el error de la muestra el $1,4 \%$ como aceptable, considerando el tamaño de la población y varianza poblacional, la validez de contenido fue realizado mediante el juicio de tres (3) expertos con experiencia en el área de emprendimiento y metodología.

La técnica de recolección de información, como uno de los ejes principales de la investigación, fue la encuesta, se elaboró el instrumento del cuestionario, para validar la "Construcción del modelo", el mismo tomó en cuenta los criterios de factibilidad curricular (cinco dimensiones), de precisión (5 dimensiones), de utilidad (4 dimensiones), de ética (3 dimensiones), de pertinencia (4 dimensiones), y contenido (4 dimensiones), cada uno de ellos con un conjunto de dimensiones en estricta vinculación con los objetivos de investigación, con opciones de respuesta dicotómicas y observaciones abiertas que a juicio de los validadores fuesen importante para mejorar la construcción definitiva del modelo.

El diseño y validación del Modelo de Gestión Teórico- Académico para el Desarrollo de Políticas y Programas de Emprendimiento Innovador en la Universidad Católica de Cuenca, desarrollados por el autor de la presente investigación, fue validado, considerando desde el punto de vista didáctico, los indicadores que tomaron en cuenta los validadores expertos, representados por la Academia, sector Gubernamental y los Sectores Productivos. Los indicadores considerados en la validación fueron la factibilidad curricular, precisión, utilidad, ética, pertinencia y contenidos.

De igual manera, Arias (2016) afirma que: "La validez del cuestionario significa que las preguntas o ítems deben tener una correspondencia directa con los objetivos de la 
investigación, es decir las interrogantes consultarán sólo aquello que se pretende conocer o medir" (p. 201).

En esta perspectiva práctica, muchas previsiones deben tomarse al momento de estimar e interpretar un coeficiente de consistencia interna. A fin de determinar la confiabilidad se aplicó el coeficiente de Alfa de Cronbach, el cual es empleado cuando el instrumento comprende ítems con alternativas de respuestas múltiples.

De igual forma, para determinar la confiabilidad del cuestionario elaborado, se procedió aplicar una prueba piloto a 15 empresas, que no forman parte de la muestra y que corresponden a la ciudad de Azogues, provincia del Cañar, todos ellos con características similares a los sujetos de este estudio, administrando una escala, de 15 ítems, a una muestra de $n=15$. De acuerdo con los resultados anteriores, se concluyó que la escala tiene una confiabilidad de consistencia interna muy alta, suficientemente alta como para ser considerada fiable. En conclusión, la confiabilidad del instrumento fue de 0,9 muy alta, así mismo, fue importante la utilización de la herramienta estadística para el desarrollo de la investigación, recurriéndose a la estadística descriptiva. El análisis de los datos es una parte fundamental de toda investigación, en tal sentido Por su parte, Hernández, Fernández, y Baptista (2016), sostiene que el procedimiento que se sigue para analizar los datos consiste en: Una vez que los datos se han codificado, transferido a una matriz, guardado en un archivo y "limpiado" los errores, el investigador procede a analizarlos utilizando un programa computacional (p.272). De allí la importancia de inspeccionar, limpiar y transformar datos con el objetivo de resaltar información útil, lo que sugiere conclusiones, y apoyo a la toma de decisiones.

En el caso concreto de la presente investigación se utilizó el software libre en español SPSS versión 26, que es una herramienta de tratamiento de datos y análisis estadístico, igualmente, para el procesamiento y análisis de los datos obtenidos a lo largo del proceso de investigación, se realizó, considerando el uso de la triangulación, validez, confiabilidad y contrastación de los instrumentos.

\section{Fundamentación Teórica.}

Abarca el marco teórico conceptual que comprende la revisión de otras investigaciones que sirvieron de antecedente a la presente investigación, se revisó la tesis doctoral de Tobar (2014), titulada: Las pequeñas y medianas empresas en Cuenca, su impacto en la economía local, presentado en la Universidad de León de España. El objetivo general fue conocer el impacto que tienen los emprendimientos en la economía de la capital azuaya, estudiar sus fortalezas y debilidades, así coadyuvar para incrementar la inversión directa del sector productivo.

Según, Urbano, (2008). Sostiene que la promoción de nuevas empresas que crean trabajo, intensifica la competitividad e incrementan la productividad a través del cambio tecnológico y la innovación. 
Es importante citar a Shane (2004), manifiestan que el emprendimiento está probado que coexiste una figura dentro del sistema económico que identifica y desarrolla oportunidades, basado en intuición y la información, rodeado de elementos de incertidumbre y riesgo, por tanto, el emprendimiento está establecido en la acción de los individuos, es por ello que se considera como plataforma solucionadora de los retos que afrontan los países.

Cuando personas tienen una idea de negocio o crea una empresa y explota una oportunidad tecnológica, estamos hablando de emprendedor tecnológico, que se centra exclusivamente en el entorno tecnológico como plataforma de contacto con el cliente tales como teleconsultas médicas, teletrabajo, entre otras, que es el que se está utilizando justamente en estas épocas del mundo COVIT. El gran reto para estos emprendedores es que sean capaces de poner en contacto a la oferta y demanda, ya que muchas veces se trata de mercados, en los que hay que explicar muy bien cuál es el beneficio que obtendrá con sus servicios. A su favor tiene exiguos costos de gestión, siempre que consiga asegurarse una buena plataforma tecnológica y centrar muy bien la idea para diferenciarla del resto. Lo más recomendable es desarrollar un buen plan de marketing digital.

Así mismo, Shane (2004), revela que los emprendedores académicos son aquellos estudiantes que han aprovechado la investigación científica y mediante esta tienen ideas de negocios y/o actividades empresariales, para la búsqueda de oportunidades con potencial de comercialización, ya sea de bienes o servicios.

Por su parte, Lee y Osteryoung (2004), con respecto a incubadoras de empresas, definen como un sistema innovador designado a asistir a emprendedores, particularmente emprendedores tecnológicos, en el desarrollo de nuevas empresas, por medio de la provisión de soporte. La incubadora apoya al emprendedor en su búsqueda de talento, tecnología, capital, y conocimiento para acelerar el desarrollo de dichas empresas y la comercialización de la tecnología.

Un tipo de emprendimiento que viene desarrollándose de manera progresiva y con mucha aceptación en los sectores académicos y sociales, en el caso de la República del Ecuador respaldado legalmente por el Plan Nacional de Desarrollo (2017-2021), por cuanto en este enfoque se le da la importancia a las políticas para el sustento de comunidades que dependen de las ideas únicas que se generan desde los cimientos del Estado.

Según Pérez (2016), una nueva forma de llevar a cabo el proceso de innovación y emprendimiento (I+D), de naturaleza cooperativa o en colaboración con los otros agentes del sistema, así como el diseño de nuevos espacios de transferencia y creación de conocimiento, orientados a la innovación en cualquiera de sus categorías o dimensiones.

Villar (2015), resalta las capacidades que favorecen la innovación, la productividad y la competitividad, y el momento para adquirir estas competencias varía entre jóvenes y adultos, si bien a ambos han de servirles para contribuir a su motivación y satisfacción en los contextos socio laborales. 
El espíritu emprendedor y la sociedad del conocimiento parten de las actitudes de las personas y se van constituyendo a lo largo de su vida, y las particularidades que el hombre tiene en forma innata se van desarrollando a medida que el individuo se va colectivizando.

En esa orientación, el hombre logrará desarrollar un espíritu emprendedor en la medida que su proceso de socialización lo conduzca en ese sentido para poder adecuarse a los cambios y transformaciones que se dan en la sociedad.

Autores como Lee y Jones (2008), analizaron los propósitos emprendedores de profesionales en el manejo de tecnologías, que abandonaron sus trabajos para iniciar una empresa.

El desarrollo local hace referencia a la capacidad que tiene una sociedad, para formular objetivos de interés colectivo y movilizar los recursos necesarios para alcanzarlos y transformar su territorio, (Alburquerque, 2004).

En América Latina y muy particularmente en el Ecuador, uno de los aspectos importantes es la migración rural urbana, experimentada en las últimas décadas, debido a la falta de oportunidades laborales, la baja calidad en la producción e ineficacia en la comercialización y la ineficiente gestión pública, generando una alta problemática socioeconómica, cultural y ambiental, Mata (2014).

La sociedad se enfrenta a fuertes presiones para el cambio, entre las cuales destacan: la globalización, el desarrollo sostenible, las nuevas tecnologías y el cambio demográfico. Una política innovadora debe reconocer y considerar tales presiones, además de mantener un enfoque global y coherente. Los países y regiones que den respuesta a estas cuestiones estarán mejor preparados para afrontar el futuro.

La innovación, para Schumpeter (2004), consistía en el dominio de emprendedores arriesgados; que, llevados por el espíritu emprendedor, querían desviar, y hasta revolucionar, la forma existente de hacer negocios. Concebía la innovación de forma amplia, incluyendo nuevas tecnologías o productos, nuevos métodos o procesos de producción, la apertura de nuevos mercados o fuentes de oferta y hasta nuevos tipos de organización o modelos de negocio. La evolución humana ha estado siempre ligada a la innovación. Los libros de texto describen las grandes innovaciones que han marcado el curso de la historia, como Internet, el automóvil o, incluso, la rueda. Ejemplos de este tipo propician que en ocasiones la innovación se asocie a la tecnología.

\section{Resultados.}

Para el procesamiento y análisis de la información de los distintos actores o participantes del presente trabajo de investigación, La tabulación e interpretación de los cuestionarios dirigidos a los ejecutivos empresariales de la provincia del Azuay, son un total de 15 ítems, que responden a (4) de tipo empresarial, (2) preguntas de gestión académica, (5) políticas de emprendimiento innovador, (4) elementos curriculares, de estas una fue tipo 
Likert, para el presente análisis se han seleccionado únicamente cinco, los más transcendentales.

En el desarrollo de su gestión empresarial, es importante la aplicación del emprendimiento.

Figura 1. Importancia del emprendimiento.

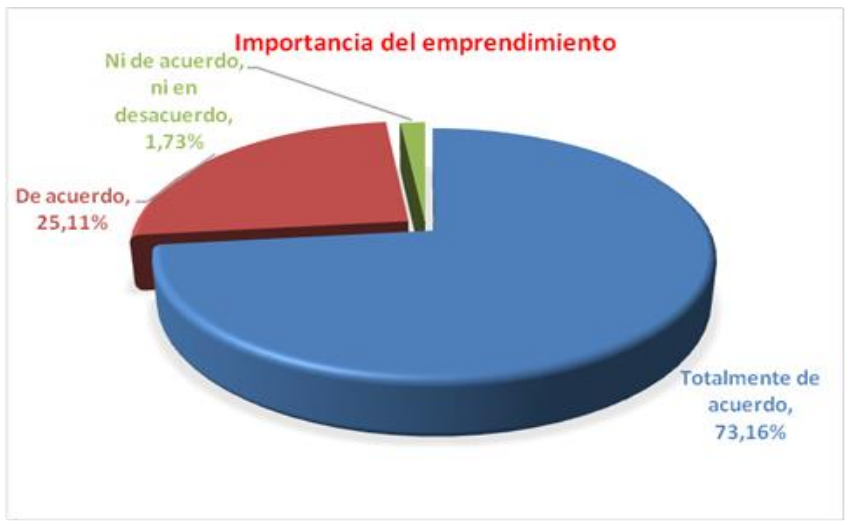

Fuente: Elaboración propia.

Con respecto al enunciado sobre el desarrollo de la gestión empresarial, si es importante la aplicación del emprendimiento, la información recogida permitió determinar que el $73,16 \%$ están de acuerdo y le dan la valía e importancia al emprendimiento.

Por otro lado, el $25.11 \%$ estuvieron de acuerdo y finalmente el $1.73 \%$ ni de acuerdo, ni en desacuerdo, esto representa una ínfima parte, ante la importancia de entender que cualquier emprendimiento que uno anhele llevar adelante requiere tiempo, paciencia, perseverancia y dedicación.

¿Cuál de las siguientes competencias dentro de la cultura emprendedora le parece importante que deberían las Universidades impartir a sus estudiantes?

Figura 2. Competencias de la cultura emprendedora.

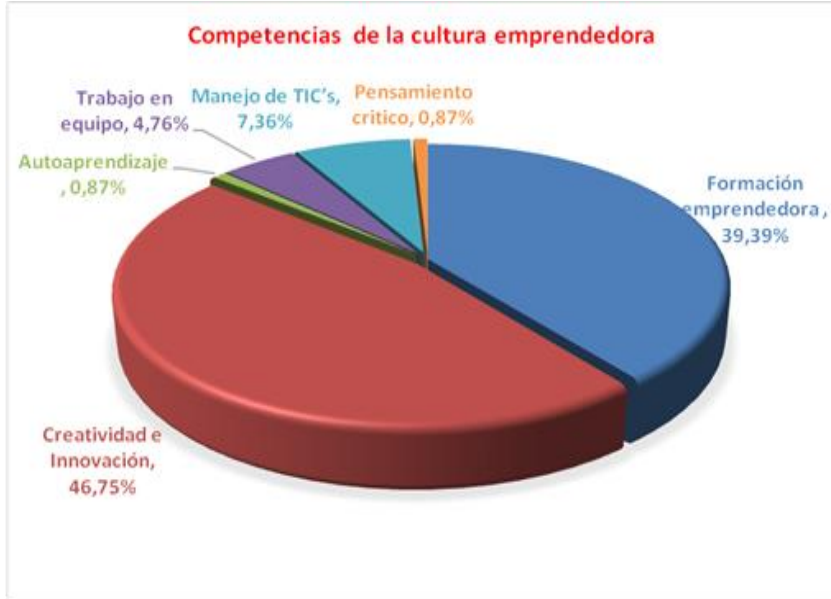

Fuente: Elaboración propia. 
Los ejecutivos empresariales encuestados asumieron en su mayoría que es importante para la sostenibilidad la creatividad e innovación empresarial, que son dos términos de los que en la actualidad se habla con bastante frecuencia, pero que realmente no se lleva a la práctica en muchas empresas. La creatividad e innovación, no sólo son valiosas y fundamentales para alcanzar el éxito empresarial, sino que servirán para lograr que una empresa pueda sobrevivir en un mercado cada vez más competitivo, según la figura $\mathrm{N}^{\circ} 2$ representa el $46,75 \%$, por tales razones, la creatividad junto con la innovación son elementos que han venido interactuando en el entorno empresarial desde hace mucho tiempo, pero que a medida que los mercados se van haciendo cada día más competitivos, dichos elementos han tomado un papel protagónico y utilizan herramientas competitivas que les permitan sostenerse con éxito en el mercado.

De la misma manera, la formación emprendedora con el 39,39\%, se ha convertido en los últimos años en una táctica social y de conocimiento para el desarrollo de habilidades que permiten en la población económicamente activa, realizar proyectos empresariales para el autoempleo con un mayor grado de certeza y posibilidad de mejorar la calidad de vida.

El 7.36\% de ejecutivos empresariales encuestados, sostuvieron que el trabajo en equipo, que permite que un grupo cohesivo multidisciplinario este articulado al paradigma de la creatividad e innovación, en donde se ponga de manifiesto el diseño de productos y procesos, administración de operaciones, marketing, recursos humanos y otros temas afines.

El análisis indico, que el $4.76 \%$ de ejecutivos encuestados indicaron que el manejo de Tics, en los mercados, hoy por hoy, reflejan la importancia de la tecnología de la información en la economía, en virtud que permiten el desarrollo de los procesos productivos innovadores de manera sostenible.

En cuanto al autoaprendizaje los empresarios encuestados determinaron en un $0.87 \%$, por ello que los empresarios manifiestan que el emprendimiento debe fomentar la capacidad en los estudiantes universitarios de resolver problemas, en la toma de decisiones y comunicación, su voluntad de asumir responsabilidades, cooperar y trabajar en red, capacidad de autoaprendizaje, proactividad e iniciativa, preparación para asumir riesgos, de allí que para la formación de futuros emprendedores, en la Universidad debe formarse futuros empresarios, trabajadores por cuenta propia, funcionarios, investigadores, docentes, altos cargos, dirigentes políticos, entre otros.

Por último, con el $0.87 \%$ de los ejecutivos empresariales encuestados sostuvieron que es importante fomentar el pensamiento crítico. Llama la atención en todos los actores encuestados la baja incidencia porcentual del pensamiento crítico.

¿Cuáles son los insumos principales que usted conoce para promover la cooperación a través de la integración productiva? 
Figura 3. Cooperación a través de la integración productiva.

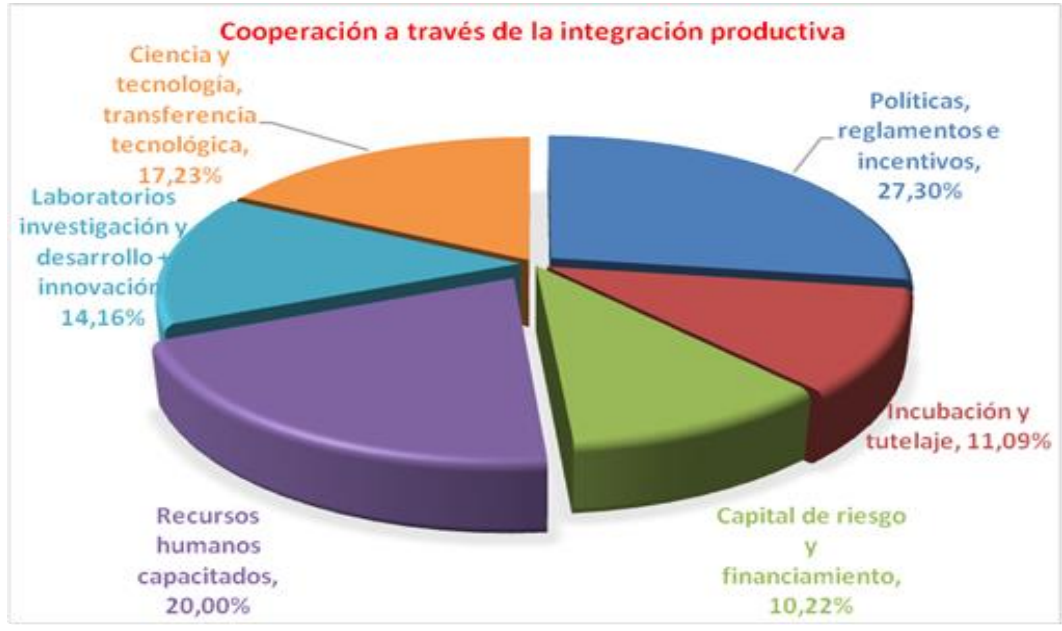

Fuente: Elaboración propia

En la pregunta sobre los insumos principales que usted conoce para promover la cooperación a través de la integración productiva, la información recogida permitió determinar, según los encuestados como se puede observar en la figura $\mathrm{N}^{\circ} 3$, la opción de las políticas, reglamentos e incentivos, es la de mayor importancia, con un porcentaje del $27,30 \%$, en tal sentido es necesario que la las UCACUE, en articulación con el gobierno nacional, concretamente con el Plan de Desarrollo Toda una Vida, trabaje con mayor énfasis en temas concernientes a las leyes, políticas, reglamentaciones y normativas para facilitar el fortalecimiento en materia de emprendimiento e innovación.

Con respecto, a los recursos humanos capacitados el análisis determinó que es la segunda considerada por los ejecutivos empresariales, con el 20\%, por tanto, es conveniente destacar que los recursos humanos es parte fundamental de toda organización y/o empresa.

Un tercer componente considerado de manera representativa, con 17,23\% de los encuestados es la transferencia de tecnología.

La información recogida permite determinar, que el $14.16 \%$ de ejecutivos empresariales encuestados manifestaron que los laboratorios de investigación y desarrollo + innovación, son importantes para la integración productiva.

En relación, a la incubación y tutelaje, el $11.09 \%$, de encuestado considero la necesidad de la creación de incubadoras y tutelaje responde a las necesidades de potenciación de las competencias en gestión del emprendimiento.

Finalmente, el $10.22 \%$ capital de riesgo y financiamiento, los ejecutivos empresariales indicaron que es importante impulsar las iniciativas de los sectores gubernamentales, con respecto a las fuentes de financiamiento, capital semilla, capital de riesgo, inversión 
ángel, Inversión de riesgo, además de formas de financiamiento para impulso de proyectos de emprendimiento.

Del detalle de las siguientes ideas, según su criterio califique a las que permiten crear valor agregado para el desarrollo de la Cátedra de Emprendimiento.

Figura 4. Crear valor agregado para el desarrollo de la Cátedra de Emprendimiento.

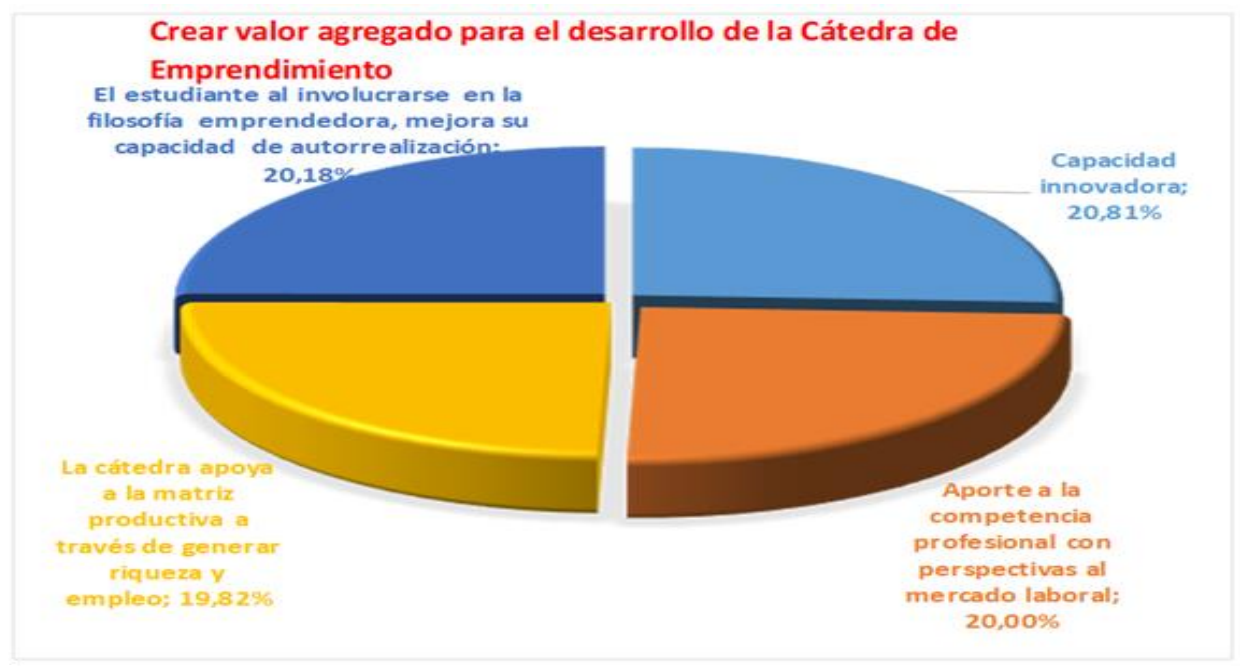

Fuente: Elaboración propia.

En cuanto a las ideas que permiten crear valor agregado para el desarrollo de la cátedra de Emprendimiento, al analizar según la figura $\mathrm{N}^{\circ} 4$, los ejecutivos empresariales encuestados consideraron el $20,81 \%$ que corresponde a la capacidad innovadora.

El 20,18\% de los empresarios encuestados al involucrarse en la filosofía emprendedora, consideraron que mejora su capacidad de autorrealización, de allí que la cultura emprendedora debe ser trabajada a largo plazo, no se puede lograr encender la chispa de la creatividad en una sociedad en la que, la política, mercados, entidades financieras, capital humano y cultura, actúan de manera dispersa, la interacción de los seis elementos proporciona el hábitat básico para desarrollar emprendimientos innovadores. Con el 20\%, los empresarios se pronunciaron con respecto al aporte a la competencia con perspectivas al mercado laboral, ya no alcanza con adquirir los conocimientos técnicos y teóricos, también es necesario tener una serie de conocimientos transversales, destrezas y aptitudes que puedan aplicarse a otros empleos y empresas y que permitan resolver los problemas profesionales de forma autónoma y flexible.

El 19,82\% considera que la cátedra de emprendimiento apoyará a la matriz productiva a través de generar riqueza y empleo. Por último, con el 19,19\% de los ejecutivos empresariales encuestados, manifestaron que la cátedra de emprendimiento aporta a elevar el nivel de conciencia social y ambiental, al respecto el cuidado del medio 
ambiente, más allá de ser una medida para combatir el deterioro del planeta, también presenta una oportunidad de negocio.

¿Cuáles de las siguientes opciones, según su criterio, favorecen la educación emprendedora?

Figura 5. Opciones que favorecen la educación emprendedora.

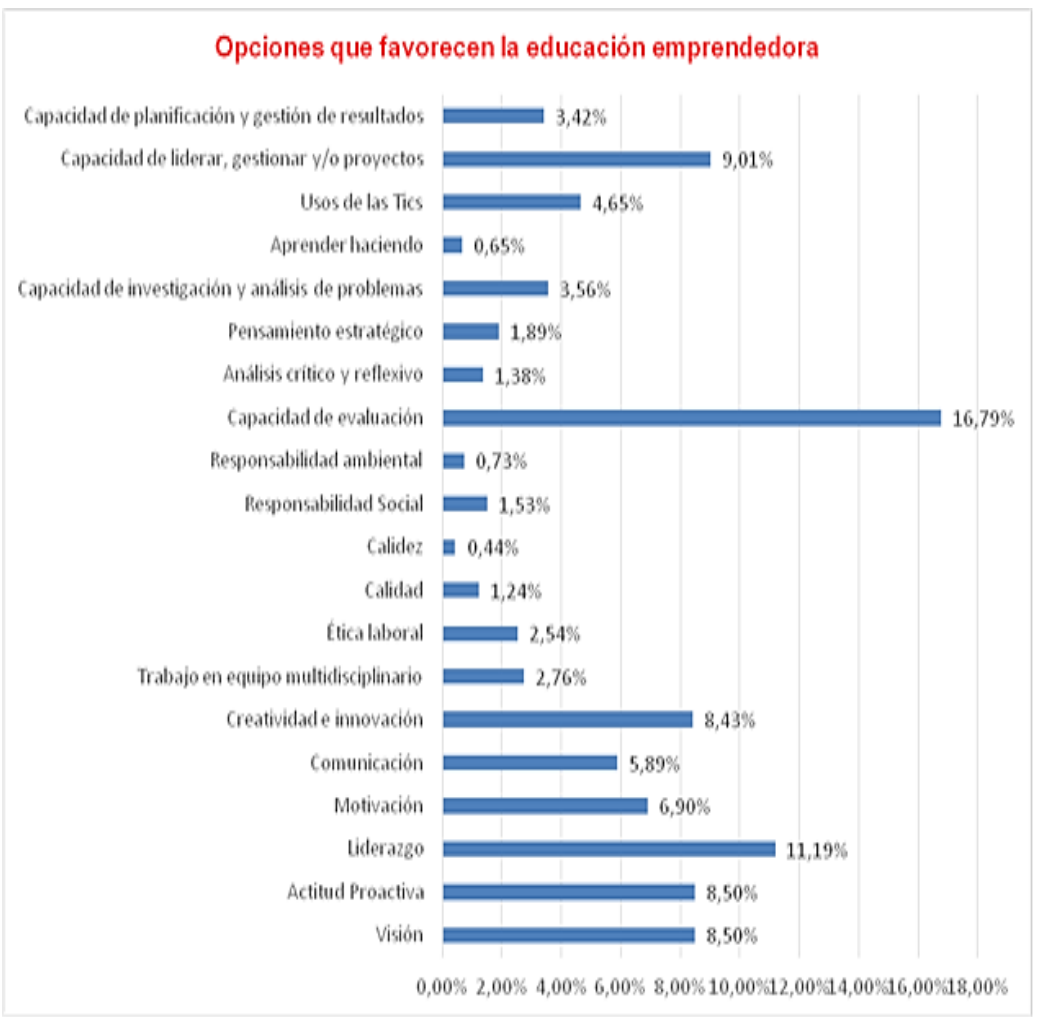

Fuente: Elaboración propia.

En cuanto a la consulta sobre las opciones que favorecen la educación emprendedora, el análisis determinó que la capacidad de evaluación que representa el 16,79\%, favorecería a la educación emprendedora.

Los resultados evidencian, que el liderazgo ayudaría a mejorar la educación emprendedora, esta consideración representa el 11,19\%, de los encuestados, es por ello que el liderazgo es la capacidad de influir positivamente para el logro de un fin valioso, común y humano, por consiguiente, la capacidad de liderazgo debe ser conocida por los alumnos, tanto para cultivarla y orientarla, como para reconocerla en otras personas, en este aspecto, el liderazgo constituye una característica básica de la competencia emprendedora: el emprendedor debe liderar e ilusionar a las personas con las que se interacciona a diario.

El 9,01\% de los ejecutivos empresariales manifestaron que es importante la capacidad de liderar y gestionar proyectos, es por ello que un gestor de proyectos debe estar suficientemente capacitado para liderar a los integrantes de su equipo de trabajo. Además, 
debe poder orientar el esfuerzo individual hacia la obtención de beneficios de carácter grupal. A su vez, debe velar por el compromiso del equipo con el proyecto a realizar.

Por su parte, con el 8,50\% los empresarios encuestados, consideraron a la visión como aquella que ayudaría a mejorar la educación emprendedora, es por ello que se ubica más allá de un objetivo común y corriente, pues es de cierto modo la razón por la que hay que organizar, planificar y trabajar duro día a día, en busca las metas planteadas, para la consecución de los beneficios y el éxito esperado.

El 8,50\%, está la actitud proactiva del emprendedor, que implica ser una persona activa, positiva, que se enfoca en hacer que las cosas sucedan y no se queda en el lamento de aquello que no funciona, sino que mientras otros se quejan, él verdadero emprendedor genera una solución y es independiente de las circunstancias.

Igualmente, los empresarios en un 8,43\% manifestaron que la creatividad e innovación fortalecerá la educación emprendedora.

Por otro lado, entre las opciones que favorecen a la cultura emprendedora el 6,90\%, consideraron la motivación emprendedora, por tanto, es un factor importante para contribuir con el desarrollo de una sociedad, por tales razones es importante el proveer instrumentos para canalizar las acciones de los emprendedores potenciales para alcanzar el éxito esperado.

Con el 5,89\% los empresarios indicaron que la comunicación es importante para impulsar la

cultura emprendedora, es bien sabido que la comunicación es la base del emprendimiento, es por ello que es importante en todos los aspectos de la vida personal, profesional y laboral.

El análisis indicó que dentro de las opciones más relevantes optadas por los empresarios encuestados se encuentra el uso de las Tics con el 4,65\%, por ello que expertos en emprendimiento y tecnologías están de acuerdo que las mismas, cumplen una importante función en el desarrollo y éxitos de emprendimiento llevados a cabo en la actualidad ya que para empezar una empresa es importante buscar información, llevar a cabo investigación y enterarse de las tendencias del mercado actual.

En menor proporción, entre las opciones que ayudarían a mejorar la educación emprendedora los ejecutivos empresariales se pronunciaron en el siguiente orden: capacidad de investigación y análisis de problemas con el 3.56 la capacidad de planificación y gestión de resultados con el 3.42\%, trabajo en equipo multidisciplinario con un $2.76 \%$, ética laboral con el $2.54 \%$, pensamiento estratégico con el $1.89 \%$, responsabilidad social con el $1.53 \%$, análisis crítico y reflexivo con el $1.38 \%$, calidad con el $1.24 \%$, responsabilidad ambiental con el $0.73 \%$, la opción aprender haciendo con el $0.65 \%$, y finalmente calidez con el $0.44 \%$. 


\section{Propuesta.}

A continuación, detallamos algunas actividades para determinar que es factible construir, un modelo de gestión teórico- académico para el desarrollo de políticas y programas de emprendimiento innovador en la Universidad Católica de Cuenca.

Es por ello que partimos del "generar ideas" más pertenece al ámbito de la creatividad e invención que de la innovación. Usualmente, los tres conceptos creatividad, invención e innovación, se relacionan y se asocian con el círculo de la investigación y el emprendimiento.

Para llevar adelante el modelo es necesario realizar las siguientes actividades, misma que se sintetizan en el grafico $\mathrm{N}^{\circ} 6$.

Figura 6. Actividades para promover el espíritu Emprendedor innovador.

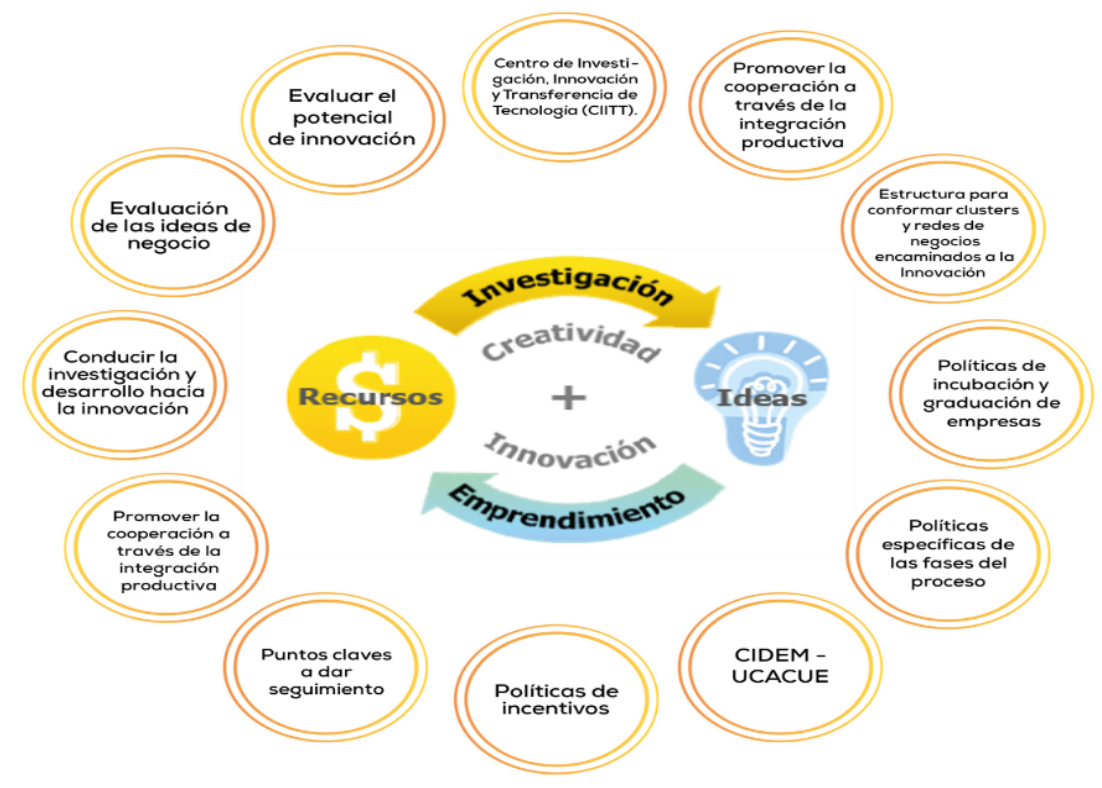

Fuente: Elaboración propia.

La Universidad Católica de Cuenca, debe dar la utilización adecuada y eficiente al Centro de Investigación, Innovación y Transferencia de Tecnología (CIITT), que tiene como objetivo constituirse en un centro de investigación de referencia a nivel nacional e internacional, haciendo uso de las mejores prácticas en el campo científico e integrando proyectos que fortalezcan la motivación y formación de los estudiantes y docentes, permitiendo la generación de emprendimientos innovadores.

Promover la cooperación a través de la integración productiva, será importante que la UCACUE, promueva e impulse el Coworking de emprendimiento, que consiste en una nueva forma de trabajo que permite a profesionales independientes, emprendedores, y MiPymes de diferentes sectores, compartir un mismo espacio de trabajo, tanto físico 
como virtual, para desarrollar sus proyectos profesionales de manera independiente, a la vez que fomentan proyectos conjuntos.

Con respecto a la estructura para conformar clusters y redes de negocios encaminados a la Innovación la articulación de varios elementos a través de la cooperación alimentada con recursos y conocimiento, es la estructura que nos permitirá desarrollar clúster y redes de negocios conformados por empresas competitivas impulsadas por el FabLab, que basa su modelo competitivo en el acceso a maquinaria tecnológica de punta, el uso de software de última generación, servicios prestados por profesionales en innovación, diseño de productos y procesos, tecnologías varias, desarrollo emprendedor y empresarial con competencias de liderazgo, trabajo en equipo, diseño de productos y procesos, administración de operaciones, marketing, recursos humanos y otros temas afines.

En relación a las políticas de incubación y graduación de empresas, será articulado, gestionado y tutelado por Centro de Incubación de Empresas Estudiantiles y Desarrollo Empresarial - CIDEM., quien permanentemente trabajará en forma conjunta con las incubadoras sectoriales, la cátedra de emprendimiento conjuntamente con los Vicerrectorados Académico y de Investigación e Innovación, Vinculación con la Sociedad y Postgrado.

En lo que respecta a las políticas específicas de las fases del proceso, el Comité de Seguimiento y Evaluación promoverá y seleccionará las ideas de negocio de conformidad a lo establecido en los criterios de selección y evaluación, considerando: Gestión productiva.

Al finalizar el primer nivel de gestión productiva, los estudiantes presentarán sus ideas de negocio; clases de emprendimiento, al finalizar el primer nivel de clases, los alumnos presentarán sus ideas de negocio; concursos de emprendimiento.

Potencial de la idea de negocio, se dará preferencia a las ideas de negocio que proporcionen una base para la innovación. Las propuestas serán pre-seleccionadas según los siguientes criterios: Potencial de innovación, mercado; y, viabilidad técnica. Valores éticos culturales y profesionales. Solo se aceptarán ideas de negocios que demuestren respetar los siguientes valores y principios: La ética profesional, la cultura de la región; y, las leyes de la República del Ecuador.

Por su parte, el criterio de evaluación se propone en el siguiente orden de criterios: Valor al cliente, tamaño del mercado objetivo, ventajas competitivas, nivel de exclusividad; y, nivel de cumplimiento, matriz de evaluación comercial y estratégica, proporciona una evaluación más detallada de una idea de negocio según los siguientes criterios: Potencial de utilidades, potencial de crecimiento, potencial de diversificación, ventaja competitiva potencial; y, riesgos estratégicos potenciales. Por su parte, al menos un socio debe tener dedicación a tiempo completo al emprendimiento. 
Contar con la aprobación del Comité de Seguimiento y Evaluación, para ello se propone considerar algunos criterios de evaluación: Análisis de factibilidad, el proyecto será factible si satisface los criterios de viabilidad en todos sus aspectos de su actuación, estos pueden ser:

Viabilidad financiera (VAN, TIR, B/C), viabilidad de mercado, viabilidad técnica, viabilidad medioambiental; y, viabilidad legal y administrativa.

En relación al CIDEM - UCACUE, durante esta fase, las empresas serán incentivadas a acelerar su crecimiento. Con este fin, se les asesorará para aumentar su competitividad, mejorar su calidad y expandir a otros mercados, para lo cual tendrá dentro de sus funciones las siguientes: Participación activa en una red de negocios o clúster, gestión de la innovación como un proceso de mejora continua, adopción de las mejores prácticas según el sector y mercado al que se dirige, cumplimiento con las normas y estándares según el sector y mercado al que se dirige; y, proceso de certificación o satisfacción de criterios de calidad de ámbito local, regional o internacional según el sector y mercado, será importante considerar los siguientes índices a lo largo del tiempo: Utilidad neta, utilidad neta / ventas, utilidad neta / patrimonio (ROE), Utilidad neta / activos totales (ROA); y, Índices de liquidez y solvencia.

En relación a las políticas de incentivos, las fuentes de financiamiento de la propuesta, es importante destacar que en la Ley Orgánica de Emprendimiento e Innovación, permite dotar a los organismos gubernamentales de capital semilla, capital de riesgo, inversión ángel, Inversión de riesgo, además de formas de financiamiento colectivo o Crowfunding, que son plataformas de financiamiento colectivo, que son recursos no reembolsables en muchos casos y sin generación de intereses que se otorgarán a proyectos de emprendimiento, que se corresponda con la Ley Orgánica de Emprendimiento e Innovación, (2020)

Así mismo, con respecto a los puntos clave, es importante dar respuesta a la siguiente interrogante: ¿Cómo conseguir los profesores y contenidos para los cursos de emprendimiento?, para dar respuesta a esta interrogante considero que es importante invitar a profesores de instituciones que son líderes en temas de emprendimiento e innovación a intercambiar experiencias comunes con la UCACUE, entre las cuales se puede citar los siguientes centros de educación superior a saber: UTPL, UPC, EAE BUSINES SCHOOL, EAN UNIVERSIDAD, STANFORD UNIVERSITY, MASSACHUSSET INSTITUTE OF TECHNOLOGY (MIT), CAMBRIDGE UNIVERSITY, OXFORD UNIVERSITY, STOCKHOLMS UNIVERSITET, entre otras). Hoy en día se puede incluso pensar en clases virtuales, por la pandemia del corona virus.

De igual manera, conducir la investigación y desarrollo hacia la innovación, para inducir la creatividad e innovación, la UCACUE debe provocar cambios en el entorno en el que se producen las actividades de investigación y desarrollo. Eso, a su vez, supone cambios culturales en muchas prácticas y actitudes convencionales, por ello es conveniente, 
establecer horarios de trabajo más flexibles, contar con áreas integradas de ocio, ejercicios y recreación, ambientes que proporcionen oportunidades de integración entre grupos, fomento de cortos periodos de actividades físicas, programación de vistas al laboratorio de fabricación digital fabLab y al CIITT.

Igualmente, evaluación de las ideas de negocio, no es nada fácil evaluar una idea de negocio cuando ni siquiera se dispone de los resultados de una encuesta o de un estudio de mercado. En este caso, se recurre a una evaluación cualitativa y se asigna notas según los criterios que se consideren apropiados, se analizará la propuesta de valor de la empresa y de cada producto o servicio que la empresa quiera colocar en el mercado, así mismo la evaluación comercial y estratégica evaluará la idea de negocio, bajo un conjunto completo de criterios, mercadeo, productividad, competitividad e internacionalización de los bienes y/o servicios.

Por último, valuar el potencial de innovación, sobre la innovación del producto, con la creación de nuevos o mera de los existentes, innovación de procesos, nuevos procesos de producción y distribución, innovación de mercado con nuevos métodos de comercialización, como en esta época de COVID el comercio electrónico por ejemplo ha tomado mucho auge.

\section{Conclusiones:}

Se explican las principales conclusiones, integrando los resultados de las encuestas a los ejecutivos empresariales y la técnica de la observación:

- La información recogida, a los ejecutivos empresariales permitió determinar que el manejo de tecnología es considerado como una de las competitividades más notables e importantes, misma que implica herramientas que hacen más fácil usar, crear, administrar e intercambiar información, la tecnología es esencial para el éxito empresarial. Los administradores y empleados dependen de ella para las operaciones del día a día.

- Referente a la Cátedra de Emprendimiento, los ejecutivos empresariales en un $49,35 \%$, revelan que se debe generar valor agregado, coinciden además la autoridades entrevistadas, en el hecho de que el estudiante al involucrarse en la filosofía emprendedora, mejora su capacidad de autorrealización, cuyo objetivo de esta materia es conocer y aplicar diversas estrategias para estimular la creatividad y la competitividad, incentivando el espíritu emprendedor que le permitan poner en práctica de manera innovadora los conocimientos adquiridos, tomando como base la solución de un problema de empleabilidad a una oportunidad de negocio.

- Existen coincidencias de los actores sociales encuestados, considerando las iniciativas, proponen que se debe desarrollar actividades académicas curriculares, mediante una simulación de negocios como una herramienta de aprendizaje 
empresarial para desarrollar la competitividad, en virtud que es una herramienta de apoyo en el proceso de aprendizaje, dado que permiten establecer un ambiente virtual de negocios a fin que los estudiantes tengan la oportunidad de participar, a través de un conjunto de decisiones, en el proceso de dirección de una empresa o de un área específica de la misma

- La información recogida permitió determinar que los emprendedores, gerentes y empresarios difícilmente dictarían materias curriculares, pues para dar clases se necesita experiencia pedagógica. Su involucramiento deberá enfocarse en las actividades extra-curriculares (mentorías, casos de estudio, conferencias, entre otras).

- Las ideas y planes de negocios de acuerdo a los empresarios, es importante para promover la creación de empresas, esto no implica una estructura complicada, información o capacitación especial para realizarlo, solo se requiere información detallada, completa y fácilmente comprensible. Un plan de negocios es necesario e importante para cualquier emprendimiento, grande o pequeño e incluso para lanzar un producto o servicio nuevo, por más pequeño que este sea. La importancia de un plan de negocios gravita en tres razones fundamentales como es la parte administrativa, de factibilidad y la financiera.

- El modelo constituye un documento teórico-metodológico que se deriva del proceso de fortalecimiento de la UCACUE y abre el camino hacia el perfeccionamiento continuo de toda su gestión y el rediseño de sus procesos; se ratifica su orientación pedagógica desde los enfoques, principios, ejes sustantivos y conceptos de la pedagogía crítica, pero enriquecidos desde la teoría de la Complejidad.

- Reforzar los procesos académicos de la UCACUE, que contribuyan a las líneas de acción propuestas en la Agenda Zonal de la Región 6, las mismas que deben basarse en la transformación de la Matriz Productiva; reducción de brechas y desigualdades socioeconómicas; $y$, sustentabilidad patrimonial.

- Fortalecer y ampliar los convenios interinstitucionales con las instituciones gubernamentales y privadas, el objetivo es promover el desarrollo de políticas públicas que faciliten y consoliden la relación entre el Estado, la academia y el sector productivo empresarial, mediante acuerdos de apoyo de organismos de desarrollo local de la región 6, cuyo objeto sea la capacitación de docentes y estudiantes y la participación conjunta, mediante el intercambio de buenas prácticas emprendedoras y propuestas de construcción de espacios de aprendizaje y debate académico.

- Se recomienda motivar a los estudiantes para que sean dueños de sus propios negocios, capitalicen sus ideas de negocios hasta alcanzar sus metas, el rol de la 
UCACUE, debe ser lograr una educación emprendedora e innovadora que permita que sus profesionales graduados se conviertan en verdaderos agentes del desarrollo local.

- Es importante destacar que existe coincidencias en el hecho de trabajar en formación emprendedora; la Universidad debe enfrentar al continuo reto de mejorar sus programas de capacitación en Emprendimiento para otorgar a los emprendedores actuales y potenciales las herramientas que necesitan los estudiantes para iniciar o hacer crecer su negocio, mediante una propuesta de valor, para lograr las mejores posibilidades de éxito de sus nuevas empresas o iniciativas o ideas empresariales, con una clara aportación al desarrollo sostenible del territorio de incidencia.

- Se recomienda la creación del proyecto de incubadora de empresas, llamado Centro de Incubación de Empresas Estudiantiles y Desarrollo Empresarial CIDEM, para lo cual se ha planteado como línea estratégica, el apoyo a la consolidación del emprendimiento académico; con pertinencia y capacidad de asesorar y acompañar eficazmente a los estudiantes, graduados y docentes de la Universidad Católica de Cuenca, en la creación y desarrollo de sus empresas que aporten al progreso, local, regional y nacional.

- El contenido de los cursos pedagógicos debe enfocarse en liderazgo, las tecnologías de la información y comunicación y los negocios, resaltando el papel de la creatividad e innovación en un ambiente competitivo, y utilizando una pedagogía que involucre trabajo en equipo y que, sobretodo, sea motivante.

\section{Referencias bibliográficas:}

Arias, F. (2016). El Proyecto de Investigación: Introducción a la metodología científica. ( $7^{\text {a }}$ Edición), Caracas - Venezuela. Editorial El pasillo, C.A.

Alburquerque, F. (2004). El Enfoque del Desarrollo Económico Local,

Organización Internacional del Trabajo, ISBN: 92-2-316549-0, (1), 2-84 2004.

Hernández Sampieri, R., Fernández-Collado, C., \& Baptista Lucio, P. (2016).

Metodología de la Investigación (Quinta Edición). México: Panamericana Formas e Impresos S.A., pág. 51.

Lee R. y Jones O. (2008). Networks, Communication and Learning during Business

Start-up: The Creation of Cognitive Social Capital. International Small Business Journal, 26 (5), 559-594.

Ley Orgánica de Emprendimiento e Innovación, (2020), Esta Ley fue publicada en el Suplemento del Registro Oficial N 151 , del 28 de febrero del 2020.

Mata, O. (2014). Los Proyectos Solidarios de Salinas de Guaranda y su Aporte para la 
Construcción de "Otra Economía". Tesis de Maestría, Facultad Latinoamericana de Ciencias Sociales, Ecuador

Pérez, L. (2016). Propuesta metodológica para la caracterización de pautas emprendedoras en materia de emprendimiento universitario.

SENPLADES. (2017). Plan Nacional para el Buen Vivir 2017-2021. Quito: SENPLADES, Gaceta oficial No 681 del 13 de julio del 2017.

Shane S. (2004). Encouraging University Entrepreneurship? The Effect of the BayhDole Acton.

Schumpeter, J. (2004).Capitalismo, socialismo y democracia. Ed. Folio. Pág. 18-124.

Tobar, L.(2014).Las pequeñas y medianas empresas en Cuenca, su impacto en la economía local, Universidad de León España, recuperado de file:/// Tesis_\%20Pequeñas\%20y\%20medianas\%20empresas\%20en\%20Cuenca.pdf

Urbano, D. (2008). Invitación al emprendimiento: Una aproximación a la creación de empresas Centros de investigación en emprendimiento. Editorial UOC.UPC. Emprendimiento (2018). Proceso de formación de empresas.

Villar, F. (2015). Los estilos de aprendizaje y su aplicación en la formación para El emprendimiento, de la Universidad de Burgos, Facultad de Educación, BurgosEspaña

Yuni, J y Urbano, C. (2006). Técnicas para investigar, segunda edición. Buenos Aires: Brujas. 


\section{PARA CITAR EL ARTÍCULO INDEXADO.}

Becerra Molina, E. (2020). El descubrimiento de oportunidades para crear valor a través del emprendimiento e innovación. Ciencia Digital, 4(3), 117-137. https://doi.org/10.33262/cienciadigital.v4i3.1305

\section{Ciencia \\ LDigital}

El artículo que se publica es de exclusiva responsabilidad de los autores y no necesariamente reflejan el pensamiento de la Revista Ciencia Digital.

El artículo queda en propiedad de la revista y, por tanto, su publicación parcial y/o total en otro medio tiene que ser autorizado por el director de la Revista Ciencia Digital.
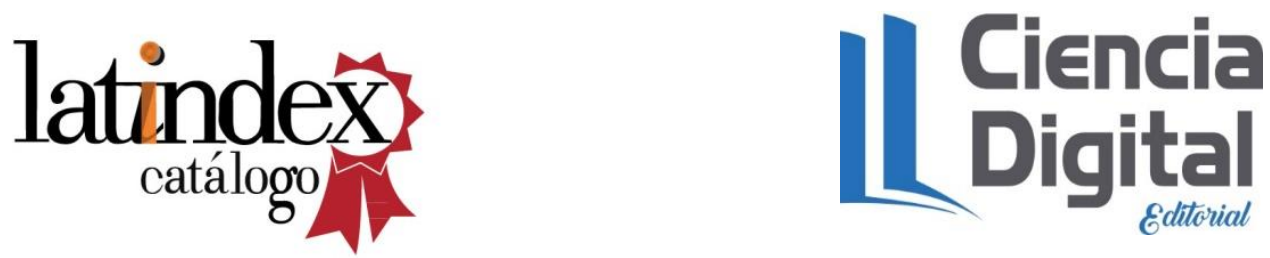